\title{
Diagnóstico preliminar de estilos de aprendizaje de los estudiantes del programa de Maestría en Gestión de Cuencas Hidrográficas de la Universidad Santo Tomás, Colombia
}

\author{
Yennifer García-M ' \\ Ligia Lugo Vargas ${ }^{2}$
}

Recibido: 04-12-2019

Aceptado: 24-06-2020

\section{Resumen}

La evaluación de estilos de aprendizaje es una manera de detectar las estrategias pedagógicas más adecuadas a utilizar en cada aula o espacio académico, para hacer del proceso de enseñanzaaprendizaje una experiencia cada vez más personalizada en la que se puedan optimizar los resultados obtenidos por los estudiantes. En esta investigación, se presentan resultados preliminares del diagnóstico de estilos de aprendizaje de estudiantes del programa de Maestría en Gestión de Cuencas Hidrográficas, a través de la aplicación del Test de Vark (Fleming y Mills, 1992), que indaga sobre cuatro de los estilos de aprendizaje más conocidos; además, se obtuvo información adicional sobre aspectos como el género, lugar de procedencia y experiencia previa en la modalidad a distancia.

\footnotetext{
1. Bióloga, M. Sc. en Biología, doctora en Ciencias - Biología. Docente del programa de Maestría en Gestión de Cuencas Hidrográficas, grupo de investigación Gestión Ambiental y de los Recursos Naturales de Colombia, Facultad de Ciencias y Tecnologías, Decanatura de División Abierta y a Distancia, Universidad Santo Tomás, Bogotá, Colombia.

Correo electrónico: yennifergarcia@ustadistancia.edu.co

ORCID: https://orcid.org/0000-0002-6741-7623

Google Académico: https://scholar.google.com/citations? user=USv_0-kAAAAJ\&hl=es

2. Bióloga con Especialización en Docencia Universitaria. M. Sc. en Evaluación en Educación. Docente del programa de Maestría en Gestión de Cuencas Hidrográficas, grupo de investigación Gestión Ambiental y de los Recursos Naturales de Colombia, Facultad de Ciencias y Tecnologías, Decanatura de División Abierta y a Distancia, Universidad Santo Tomás, Bogotá, Colombia.

Correo electrónico: ligialugo@ustadistancia.edu.co

ORCID: https://orcid.org/0000-0002-2560-8966

Google Académico: https://scholar.google.es/citations?user=1QxGJsYAAAAJ\&hl=es
} 
Se espera, con este diagnóstico preliminar, identificar cuáles son las principales estrategias que favorecen a los estudiantes, para fortalecer el proceso educativo en este y otro tipo de programas de modalidad a distancia.

Palabras clave: estilos de aprendizaje, Maestría en Cuencas Hidrográficas, educación a distancia, Test de Vark.

\section{Preliminary diagnosis of learning styles of students of the Master's Program in Watershed Management at the Universidad Santo Tomás, Colombia}

\section{Abstract}

The evaluation of learning styles is a way of detecting the most appropriate pedagogical strategies to be used in each classroom or academic space, to make the teaching-learning process an increasingly personalized experience in which the results obtained by students can be optimized. In this research, preliminary results of the diagnosis of learning styles of students of the Master's Program in River Basin Management are presented, through the application of the Vark Test (Fleming and Mills, 1992), which investigates four of the styles of best known learning; In addition, additional information was obtained on aspects such as gender, place of origin and previous experience in distance learning. It is expected, with this preliminary diagnosis, to identify which are the main strategies that favor students, to strengthen the educational process in this and other types of distance learning programs.

Keywords: learning styles, Master in Watersheds, distance education, Vark Test. 


\section{Introducción}

De acuerdo con Martínez y Briones (2007), durante varios años fueron desarrollados distintos sistemas de tutoría educativa, definida como una actividad de acompañamiento, seguimiento y apoyo al estudiante en el proceso de personalización del aprendizaje. Las universidades anglosajonas fueron las pioneras en el desarrollo del sistema de tutorías personalizadas o individualizadas para asistir al estudiante en su formación académica, personal y social. Ese sistema se caracteriza por una relación entre el tutor y el estudiante basada en una supervisión del aprendizaje por medio de procesos evaluativos, con la participación del estudiante (Martínez y Briones, 2007). Como es una relación de ayuda para el aprendizaje requiere una relación interpersonal estudiante-tutor, y una "pedagogía de acompañamiento", en palabras de Martínez y Briones (2007).

Todas las personas aprenden de formas variadas, teniendo en cuenta los sentidos que son considerados más útiles para recibir, procesar y responder ante la información proveniente del medio; por esta razón en un salón de clase es posible encontrar estudiantes que captan las ideas y entienden fácilmente con escuchar una única vez, otros necesitan imágenes o mapas mentales y otros aprenden mejor de manera autónoma. Estas situaciones se dan porque, a medida que se avanza en un proceso de aprendizaje, se desarrollan diversos estilos (Centro de Recursos para el Éxito Estudiantil [CREE], 2014). Una manera de mejorar el proceso de los estudiantes es diagnosticar sus diversos estilos de aprendizaje para detectar las estrategias pedagógicas más adecuadas en cada aula, haciendo del aprendizaje una experiencia cada vez más personalizada. Para realizar este diagnóstico puede recurrirse a la aplicación de encuestas.

Este artículo presenta un diagnóstico preliminar de los diversos estilos de aprendizaje de un grupo de estudiantes del programa de Maestría en Gestión de Cuencas Hidrográficas de la Facultad de Ciencias y Tecnologías, División de Educación Abierta y a Distancia, de la Universidad Santo Tomás. Este programa académico, de 
modalidad abierta y a distancia, inició con su primera cohorte en el segundo semestre de 2017. Surgió ante la necesidad de abordar la gestión del recurso hídrico a partir de las cuencas hidrográficas, para reconocer las múltiples funciones del agua en contextos urbanos y rurales, y en respuesta a la importancia de analizar los impactos del agua como líquido vital, con el fin último de preservar la vida en la tierra (Maestría en Gestión de Cuencas Hidrográficas, 2015). La acreditación institucional fue otorgada por el Ministerio de Educación Nacional mediante la Resolución n. 9264 del 18 de octubre de 2011, y su norma interna de creación es el Acuerdo 14 del 28 de abril de 2014 del Consejo Superior de la Universidad Santo Tomás. La duración del programa es de cuatro periodos semestrales, con un total de 50 créditos, con sede principal en Bogotá. Gracias a su modalidad, tiene presencia en distintas regiones del país e imparte sus clases a través del uso de plataformas virtuales y herramientas como Moodle, para promover la autonomía y personalización del aprendizaje. El perfil ocupacional de los egresados está orientado hacia niveles directivos o ejecutivos, donde puedan ejercer liderazgo y formar empresa. Además, los egresados deben tener capacidad de hacer análisis espacial, realizar investigación interdisciplinar básica o aplicada y desarrollar modelos de gestión operativa multidimensional, públicos o privados (Maestría en Gestión de Cuencas Hidrográficas, 2015).

Para el desarrollo de esta investigación se empleó el Test Vark, formato en un archivo Excel creado por José Alfredo Martínez Valdez, a partir de los planteamientos de Neil Fleming y Collen Mills (1992), para la clasificación de las personas de acuerdo con su preferencia en la modalidad sensorial a la hora de procesar información o contenidos educativos. Esta herramienta se aplicó para conocer cómo trabaja el estudiante con la información y cuál es su estilo de aprendizaje preferido cuando debe captar y procesar ideas y proporcionar información.

El diagnóstico preliminar desarrollado permitió identificar que los estilos auditivo y kinestésico son los principales tipos de aprendizaje, por lo cual es recomendable el uso de tutoriales con audio e instrucciones verbales, grupos de discusión, chats y exposiciones. 
Además de estos recursos pedagógicos, se recomienda el uso de simulaciones, videos y demostraciones, que constituyen los principales recursos de la educación a distancia. Este resultado confirma que esta modalidad de educación favorece más a aquellos estudiantes que poseen la capacidad de captar información a partir de herramientas visuales y el desarrollo de prácticas virtuales(Gómez, Ostos, Gallego, \& García, 2019).

\section{Metodología}

Se aplicó el Test Vark, formato en un archivo Excel creado por José Alfredo Martínez Valdez, a partir de los planteamientos de Neil Fleming y Collen Mills (1992), que clasifica los estilos de aprendizaje en cuatro principales: visual $(V)$, auditivo $(A)$, kinestésico $(K)$ y lectoescritor $(R)$, y permite identificar situaciones de aprendizaje que el estudiante puede aprovechar a su favor. La encuesta fue aplicada a 30 estudiantes del programa en mención, en el primer periodo académico del 2019. En las encuestas se recopiló información adicional como género, rango de edad, modalidad en que cursaron estudios previos (presencial o a distancia) y lugar de procedencia. Los datos obtenidos fueron organizados y procesados en el programa Excel para obtener los resultados preliminares que son presentados en términos de porcentaje.

\section{Resultados}

\section{Caracterización de la población}

El $57 \%$ de los estudiantes son hombres y el $43 \%$ mujeres; sus edades son diversas, con estudiantes entre 21 y 58 años de edad: el 37 $\%$ se encuentra entre los 31 y 40 años, $30 \%$ entre 41 y 50 años, 23 $\%$ entre 26 y 30 años de edad, $7 \%$ con edades de 52 y 58 años y un estudiante con 21 años que representa el $3 \%$ (figura 1). 
Figura 1. Distribución de género $(A)$ y rangos de edad $(B)$ de los estudiantes de la Maestría en Gestión de Cuencas Hidrográficas.



Figura $1 \mathrm{~A}$.

\section{Rangos de edad}
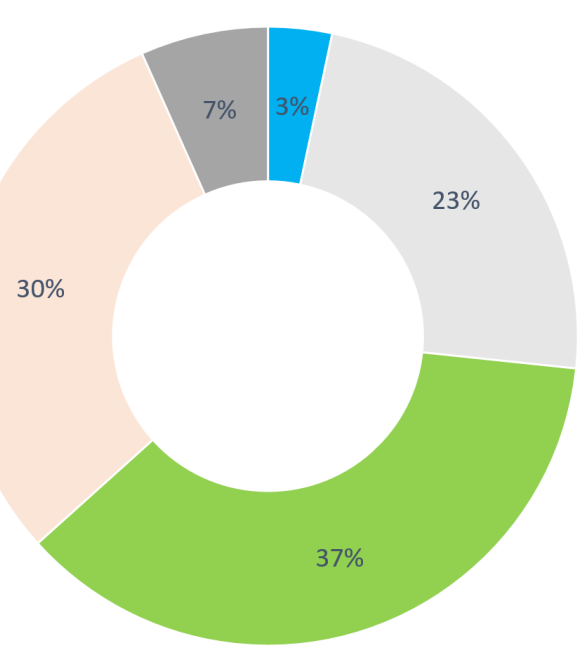

$26-30$

$=31-40$

$41-50$

- $52-58$

Figura 1B.

Fuente: elaboración propia. 
Como el programa se imparte en modalidad a distancia cuenta con estudiantes de distintas regiones del país: de los 30 encuestados, 14 se encuentran en el departamento de Cundinamarca (10 son de Bogotá y 1 en cada uno de los siguientes municipios: Anapoima, Soacha, Ubaté y Zipacón), 3 del departamento de Nariño (Pasto), 3 del departamento del Valle (Cali), 1 proveniente del departamento del Tolima, de Espinal; los demás estudiantes se encuentran distribuidos en otras ciudades como Mocoa (Putumayo), Sogamoso (Boyacá), Villavicencio (Meta), Valledupar (Cesar), Cerrito (Santander), Ocaña (Norte de Santander), Quibdó (Chocó), Montería (Córdoba) y Arauca (figura 2A).

Cuando se consultó a los estudiantes encuestados si tenían experiencia previa en estudios de modalidad a distancia, se encontró que el $53 \%$ sí la tiene, mientras el $47 \%$ no (figura 2B). La mayoría de los estudiantes que sí tienen una experiencia previa de educación a distancia provienen del programa de Especialización en Ordenamiento y Gestión de Cuencas Hidrográficas de la misma facultad.

Figura 2. Principales departamentos de proveniencia (A) y experiencia en educación virtual o a distancia previa (B) de los estudiantes del programa de la Maestría en Gestión de Cuencas Hidrográficas.

\section{Departamentos}

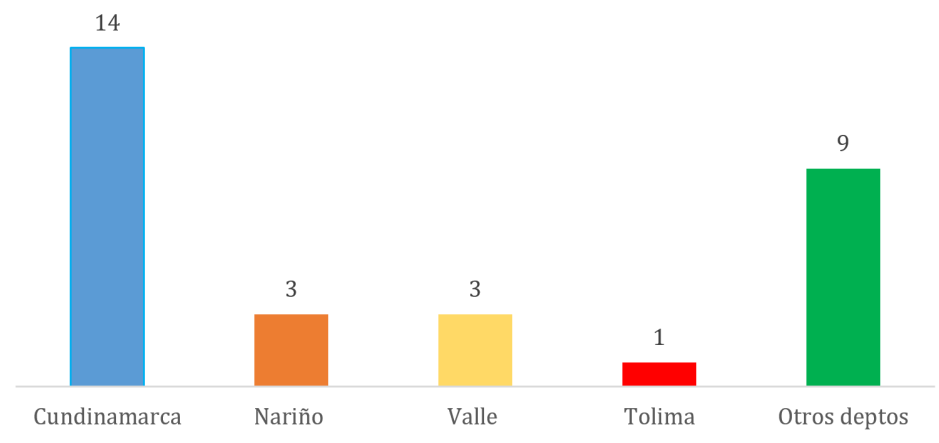

Figura 2A. 


\section{Educación virtual o a \\ distancia previa}

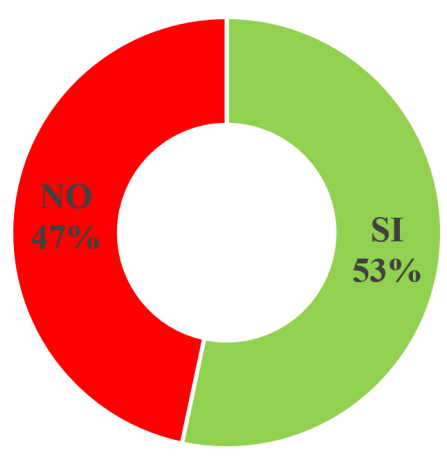

Figura 2B.

Fuente: elaboración propia.

\section{Diagnóstico de los estilos de aprendizaje}

En la población encuestada se encontró que predomina el estilo de aprendizaje auditivo ( $\mathrm{A}, 40 \%)$, seguido por el estilo kinestésico ( $\mathrm{K}$, $30 \%)$; y en menor proporción se encuentran los estilos lectoescritor ( $R, 14 \%)$ y visual (V, $10 \%)$ (figura 3 ).

Figura 3. Estilos de aprendizaje de los estudiantes de la Maestría en Gestión de Cuencas Hidrográficas.

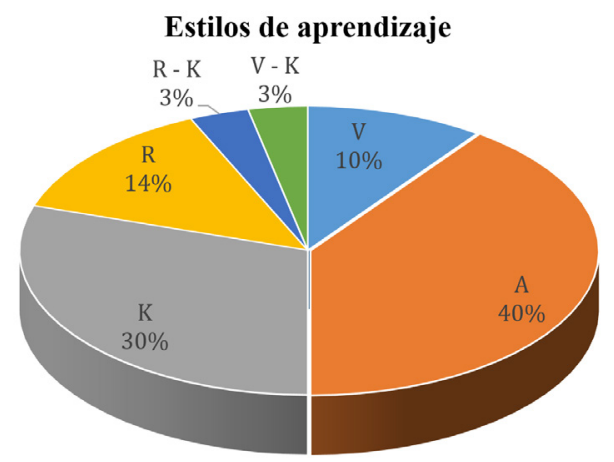

Fuente: elaboración propia. 


\section{Discusión}

Los resultados preliminares de esta investigación permiten evidenciar cómo la educación a distancia toma cada vez mayor fuerza gracias al desarrollo de las nuevas tecnologías que brindan la posibilidad de que casi cualquier persona, de diferente género, rango de edad y procedencia, pueda adquirir competencias profesionales. En el caso particular del programa de la Maestría en Gestión de Cuencas Hidrográficas es posible observar que actualmente se cuenta con una variedad de estudiantes, entre hombres y mujeres con edades entre 21 y 58 años, que provienen de distintas regiones del país. De acuerdo con García (2017), la educación a distancia constituye un proceso de enseñanza-aprendizaje novedoso porque, por un lado, fomenta el aprendizaje autónomo de los estudiantes, pero también el aprendizaje colaborativo y cooperativo, en donde los mismos estudiantes aprenden con otros, de otros y para otros, a través de plataformas virtuales y entornos de aprendizaje(Gómez, 2019; Ostos-Ortiz \& AparicioGómez).

Esta modalidad de educación tiene ventajas como la posibilidad de que una misma institución organice una amplia oferta de cursos sin concentración geográfica única, como una respuesta a la mayoría de las actuales necesidades de formación (García, 2017). Además, esta modalidad educativa posee flexibilidad porque permite desarrollar el estudio sin los requisitos rígidos de espacio, asistencia o tiempo, propios del modelo educativo tradicional (García, 2017), y permite establecer un equilibrio entre las demandas familiares, laborales o de formación. Además, el estudiante se convierte en el centro del proceso de aprendizaje porque, entre otras cosas, se propicia la autoevaluación de los aprendizajes, y porque la formación puede ser conectada casi inmediatamente con el trabajo, que en el caso de un nivel de maestría pretende mejorarse (García, 2017). Finalmente, es un tipo de educación inclusiva porque abre la oportunidad a sectores sociales que poseen dificultades para acceder a la educación en el modelo tradicional, ya sea por falta de tiempo, o por imposibilidad de desplazamiento hasta el centro educativo. Como parte de la 
visión del estudiante como centro del aprendizaje, Alonso, Gallego y Honey (2004), citados por Gutiérrez-Tapias (2018), mencionan que conocer la predominancia de los estilos de aprendizaje que tienen los estudiantes es fundamental para fortalecer la acción pedagógica y elevar los niveles de calidad educativa y el rendimiento académico. En este sentido, los estilos de aprendizaje son nociones importantes que los docentes deben conocer, porque repercuten en su forma de enseñar.

La Maestría en Gestión de Cuencas Hidrográficas, en su modalidad a distancia, favorece los estilos de aprendizaje principales detectados en este diagnóstico preliminar, auditivo y kinestésico, debido a que permite y promueve el uso de estrategias para mejorar la experiencia de aprendizaje de los estudiantes como:

- Uso de imágenes, infografías, mapas conceptuales, herramientas audiovisuales para la introducción y el desarrollo de un tema en particular.

- Exposiciones por parte de los estudiantes, con una estructura lógica determinada, para que tengan que organizar sus ideas y extraer información relevante.

- Foros de discusión que permiten la libre presentación de ideas individuales para producir nuevas soluciones.

- Prácticas, que pueden ser virtuales, en las cuales los estudiantes aplican un conocimiento específico en un entorno virtual, que les entrega una experiencia casi real de estudios de caso. En este aspecto, el programa inició, en el segundo semestre del 2019, la implementación de prácticas virtuales, aprovechando el estilo de aprendizaje principal de los estudiantes (kinestésico), para mejorar su experiencia y fortalecer su perfil ocupacional, utilizando el método de análisis de casos, asociados con las principales temáticas relacionadas con la gestión del recurso hídrico. 
- Uso de software estadísticos, para que el estudiante maneje, programe e interprete resultados de análisis de sus proyectos de investigación.

Todas estas estrategias constituyen una forma de fortalecer el aprendizaje autónomo, pero también el aprendizaje cooperativo y colaborativo, propios de la modalidad a distancia. Sin embargo, cada uno de los estilos de aprendizaje descritos en el test usado en este estudio presenta estrategias pedagógicas variadas, que pueden ser aprovechadas por el docente para fortalecer el proceso formativo de sus estudiantes.

Un estilo de aprendizaje importante es el lectoescritor, aunque, como se mencionó, no es uno de los que predomina. Es necesario promover y fortalecer hábitos de lectura y escritura en los estudiantes para mejorar el desarrollo de los proyectos de investigación, como es el caso del trabajo de grado, requisito para optar al título de magíster en Gestión de Cuencas Hidrográficas, pues deben plantear un problema de investigación y buscar una solución. Esto requiere que recopilen y analicen información para preparar un documento escrito. Comprender y producir textos es un proceso que requiere un alto nivel cognitivo, en particular una tesis de maestría constituye un documento que muestra la estrecha relación entre escritura e investigación, y el texto escrito es el reflejo del proceso investigativo (Ochoa, Gordillo y Castro, 2018). Por esta razón, la lectura y la escritura es un componente importante que no puede dejarse de lado en el proceso de formación de un estudiante de cualquier nivel educativo, especialmente en un nivel de maestría. De esta forma, es necesario enseñar a los futuros maestrantes buenas competencias de lectura y escritura, que mejorarán su desempeño laboral y su perfil ocupacional.

\section{Conclusiones}

Un diagnóstico preliminar de los estilos de aprendizaje permite mejorar estos procesos de los estudiantes, de tal manera que los 
contenidos y las estrategias pedagógicas se hagan de manera casi personalizada, teniendo en cuenta las fortalezas de los estudiantes a la hora de aprender, para elevar los niveles de calidad educativa y el rendimiento académico de los estudiantes. Por supuesto, dicha personalización de las estrategias fortalece las habilidades pedagógicas de cada uno de los tutores y docentes.

Aunque el estilo lectoescritor no predomina en la población analizada, es necesario fortalecer este tipo de habilidades para mejorar su perfil profesional y atender a su perfil ocupacional.

\section{Recomendaciones}

Vale la pena consultar otro tipo de estrategias pedagógicas, además de las mencionadas en este estudio, y socializarlas con los estudiantes y el equipo docente para fortalecer el proceso pedagógico de aprendizaje.

\section{Referencias bibliográficas}

Centro de Recursos para el Éxito Estudiantil (CREE). (2014). ¿Por qué no todo se aprende igual? Estilos de aprendizaje. Gimnasio académico, rutinas para ejercitar tu cerebro. Barranquilla: Universidad del Norte.

Fleming, N. y Mills, C. (1992). Modelo Vark: sistemas de representación. Recuperado de https://es.scribd.com/document/357747124/TESTVARK-LIBRE-xIsx

García, L. (2017). Educación a distancia y virtual: calidad, disrupción, aprendizaje adaptativo y móvil. Revista Iberoamericana de Educación a Distancia, 20(2), 9-25. Recuperado de http://revistas.uned.es/index. $\mathrm{php} / \mathrm{ried} /$ article/view/18737 
Gómez, O. Y. A. (2019). Uso y apropiación de las TIC en educación. Revista Interamericana de Investigación, Educación y Pedagogía, RIIEP, 12(1), 253-284.

Gómez, O. Y. A., Ostos, O. L., Gallego, M. A. C., \& García, C. A. (2019). Análisis sistemático de los artículos publicados en la revista interamericana de educación, investigación y pedagogía (RIIEP) durante 2014 a 2019. Revista Interamericana de Investigación, Educación y Pedagogía, RIIEP, 12(2), 225-240.

Gutiérrez-Tapias, M. (2018). Estilos de aprendizaje, estrategias para enseñar: su relación con el desarrollo emocional y "aprender a aprender". Tendencias Pedagógicas, 31, 83-96. Recuperado de https://repositorio. uam.es/handle/10486/680833

Maestría en Gestión de Cuencas Hidrográficas (2015). Documento Maestro. Facultad de Ciencias y Tecnologías. Universidad Santo Tomás. Bogotá, Colombia.

Martínez, M. T. y Briones, S. (2007). Contigo en la distancia, práctica tutorial en entornos formativos virtuales. Pixel-Bit. Revista de Medios y Educación, 29, 81-86. Recuperado de https://idus.us.es/ handle/11441/45623

Ochoa, L., Gordillo, A. y Castro S. M. (2018). Lectura, escritura e investigación en la educación superior. Bogotá: Universidad Distrital Francisco José de Caldas.

Ostos-Ortiz, O.-L., \& Aparicio-Gómez, O.-Y. Investigación, innovación y transferencia del conocimiento.

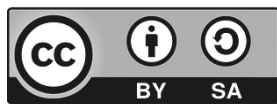

